\title{
Magnetic resonance imaging of the hand joints in patients with inflammatory bowel disease and arthralgia: a pilot study
}

\author{
LKPM Brakenhoff ${ }^{1 *}$, W Stomp ${ }^{2 *}$, FA van Gaalen ${ }^{3}$, DW Hommes ${ }^{1,4}$, JL Bloem² $^{2}$ DMFM van der Heijde ${ }^{3}$, HH Fidder ${ }^{5}$, \\ M Reijnierse ${ }^{2}$
}

Departments of ${ }^{1}$ Gastroenterology and Hepatology, ${ }^{2}$ Radiology, and ${ }^{3}$ Rheumatology, Leiden University Medical Centre, The Netherlands, ${ }^{4}$ Center for Inflammatory Bowel Diseases, UCLA Health System, Los Angeles, CA, USA, and ${ }^{5}$ Department of Gastroenterology and Hepatology, University Medical Centre Utrecht, The Netherlands

Objectives: To assess whether subclinical inflammatory changes are present on magnetic resonance imaging (MRI) in patients with inflammatory bowel disease (IBD) and arthralgia.

Method: In this pilot study, painful hand joints [metacarpophalangeal (MCP), proximal interphalangeal (PIP), and/or distal interphalangeal (DIP)] of 11 IBD patients (age 18-45 years) with continuous pain for $>6$ weeks were scanned on a 1.5-T extremity MRI system. A control group of 11 IBD patients without joint pain who were matched for type and disease duration of IBD, gender, and age was included. All patients were clinically examined by a rheumatologist for the presence of pain and arthritis. Imaging was performed according to a standard arthritis protocol with intravenous contrast administration on the same day. Images (blinded for clinical information) were evaluated by two readers in consensus for the presence of joint fluid, synovitis, tenosynovitis, enthesitis, erosions, cartilage defects, and bone marrow oedema.

Results: Enthesitis was seen in three hand joints (MCP 2, MCP 3, PIP 3) of 2/11 (18\%) arthralgia patients and in none of the control group $(\mathrm{p}=0.48)$. A small amount of subchondral bone marrow oedema was seen in the metacarpal head of two controls. No other abnormalities were observed.

Conclusions: Several young IBD patients with chronic hand pain had subclinical inflammation on MRI, which invites for further study in a larger group of patients.

Inflammatory bowel disease (IBD), Crohn's disease (CD), and ulcerative colitis (UC) are associated with a variety of extraintestinal manifestations of which articular involvement is the most common, with a prevalence of $16-33 \%$ (1). Joint pain can be caused by inflammation (arthritis, enthesitis), but in many IBD patients no inflammation is found (arthralgia). Arthralgia could be the result of subclinical inflammation and magnetic resonance imaging (MRI) is a sensitive imaging technique that may identify inflammation that is not yet clinically apparent (2). The aim of our pilot study was therefore to assess whether inflammatory changes could be detected on MRI in IBD patients with painful hand joints without clinically active arthritis or enthesitis.

Lianne KPM Brakenhoff, Department of Gastroenterology and Hepatology, Leiden University Medical Center, PO Box 9600, 2300 RC Leiden, The Netherlands

E-mail: k.p.m.brakenhoff@lumc.nl.

* LKPM Brakenhoff and W Stomp share the first authorship.

Accepted 8 January 2014

\section{Method \\ Patients}

Eleven patients with arthralgia of the small joints of the hand for more than 6 weeks (without clinically active arthritis/enthesitis) and with a pain score of $\geq 4$ on an 11point numerical rating scale (NRS) were recruited from the Gastroenterology and Hepatology outpatient clinic. All patients had endoscopically or histologically proven $\mathrm{CD}$ or UC for at least 3 months. IBD patients aged between 18 and 45 years were selected to reduce the likelihood of the coexistence of osteoarthritis. A control group of 11 IBD patients without joint pain who were matched for type and disease duration of IBD, gender, and age was included.

The most painful joint was imaged; if joints on both the left and right side were equally affected then the dominant side was imaged. Radiographs were acquired to exclude patients with an explanation for peripheral joint pain on X-ray. The study was approved by the local medical ethics committee and all patients provided written informed consent.

\section{Imaging}

MRI examinations were performed on an MSK Extreme 1.5-T extremity MRI scanner (GE Healthcare, Wilmington, 
MA, USA). A single region of the (most) painful joints [both metacarpophalangeal (MCP) and proximal interphalangeal (PIP) or PIP and distal interphalangeal (DIP)] was imaged. Coronal T1-weighted, coronal T2-weighted with fat suppression, and coronal and axial $\mathrm{T} 1$-weighted images with fat suppression after gadolinium (Gd)-chelate intravenous injection were acquired.

MRI parameters evaluated were the presence of joint fluid, synovitis, tenosynovitis, enthesitis, erosions, cartilage defects, and bone marrow oedema. Other intraarticular pathology was ruled out as a possible cause for the pain. MRI images were evaluated in consensus by two musculoskeletal radiologists (MR and JLB) blinded to the patients' complaints.

\section{Statistical analysis}

Comparisons between patients and matched controls were performed using Mann-Whitney tests, Pearson's $\chi^{2}$ test, or Fisher's exact tests as appropriate.

\section{Results}

Between May 2011 and August 2012, 11 IBD patients with arthralgia of the small joints of the hand and 11 IBD patients without arthralgia were included (Table 1). No patients were excluded based on their clinical examination or radiographs. Type of IBD, age, gender, IBD disease duration, and age at onset of IBD did not significantly differ between the patients with and without arthralgia. None of the patients fulfilled the Assessment of SpondyloArthritis International Society (ASAS) classification criteria of spondyloarthritis (SpA) or had been previously diagnosed with another inflammatory joint disease.

The painful joints in the 11 IBD patients with hand arthralgia (eight right, three left, median number of painful joints $=5$, range $2-14)$ were: $\mathrm{MCP}(\mathrm{n}=29)$, PIP $(n=34)$, and DIP $(n=5)$. MRI included either MCP (2-5) and PIP (2-5) or PIP (2-5) and DIP (2-5) joints. In total, 53 (MCP, PIP, and DIP) painful hand joints were scanned.

Minimal enthesitis was seen in two of the $11(18.2 \%)$ IBD patients with arthralgia, but in none of the control group $(p=0.48)$. Enthesitis was found at the radial side of the second and third MCP joints in one patient and in another arthralgia patient at the radial side of the third PIP joint (Figure 1). Enthesitis corresponded to painful joints in the arthralgia patients.

Bone marrow oedema was not found in the arthralgia patients, but a small amount of subchondral bone marrow oedema was seen in the metacarpal head of two $(2 / 11,18.2 \%, p=0.48)$ controls. Joint fluid, synovitis, tenosynovitis, and cartilage defects were absent in both groups.

\section{Discussion}

We investigated the presence of subclinical inflammation on MRI in the painful small joints of the hands (MCP, PIP, and DIP joints) of 11 IBD patients with arthralgia without clinically active arthritis or enthesitis. Enthesitis was detected in two of these patients.

Table 1. Patient demographics and disease characteristics.

\begin{tabular}{|c|c|c|}
\hline & $\begin{array}{l}\text { IBD with arthralgia } \\
\qquad \mathrm{n}=11\end{array}$ & $\begin{array}{l}\text { IBD without arthralgia } \\
\qquad \mathrm{n}=11\end{array}$ \\
\hline $\mathrm{CD} / \mathrm{UC}, \mathrm{n}(\%)$ & $10(90.9) / 1(9.1)$ & $10(90.9) / 1(9.1)$ \\
\hline Age (years), mean $\pm S D$ & $38.3 \pm 5.5$ & $40.5 \pm 4.9$ \\
\hline Sex male, $\mathrm{n}(\%)$ & $3(27.3)$ & $3(27.3)$ \\
\hline Age of onset of arthralgia (years), mean $\pm S D$ & $32.5 \pm 7.0$ & - \\
\hline Duration of arthralgia (years), mean $\pm S D$ & $5.3 \pm 5.7$ & - \\
\hline \multicolumn{3}{|l|}{ Arthralgia coincides with relapses of IBD, $n(\%)$} \\
\hline Yes & $4(36.4)$ & - \\
\hline No & $6(54.5)$ & - \\
\hline Unknown & $1(9.1)$ & - \\
\hline Joint pain 11-point NRS, median (IOR) & $5.0(4-7)$ & - \\
\hline Onset of arthralgia before IBD diagnosis, $n(\%)$ & $4(36.4)$ & - \\
\hline \multicolumn{3}{|l|}{ Arthralgia medication, $\mathrm{n}(\%)$} \\
\hline COX-2 inhibitor & $2(18.2)$ & - \\
\hline Disease duration of IBD (years), mean $\pm S D$ & $10.5 \pm 7.6$ & $14.3 \pm 4.9$ \\
\hline Age of onset of IBD (years), mean $\pm S D$ & $27.2 \pm 8.8$ & $25.9 \pm 6.0$ \\
\hline \multicolumn{3}{|l|}{ IBD medication, $\mathrm{n}(\%)$} \\
\hline Anti-TNF (infliximab/adalimumab) & $4(36.4)$ & $6(54.5)$ \\
\hline Thiopurines (azathioprine/thioguanine/6-mercaptopurine) & $3(27.3)$ & $4(36.4)$ \\
\hline Mesalazine & $1(9.1)$ & $1(9.1)$ \\
\hline Corticosteroids & $2(18.2)$ & - \\
\hline None & $1(9.1)$ & $3(27.3)$ \\
\hline
\end{tabular}

IBD, Inflammatory bowel disease; CD, Crohn's disease; UC, ulcerative colitis; SD, standard deviation; NRS, numerical rating scale; IQR, interquartile range; COX, cyclooxygenase; TNF, tumour necrosis factor. 


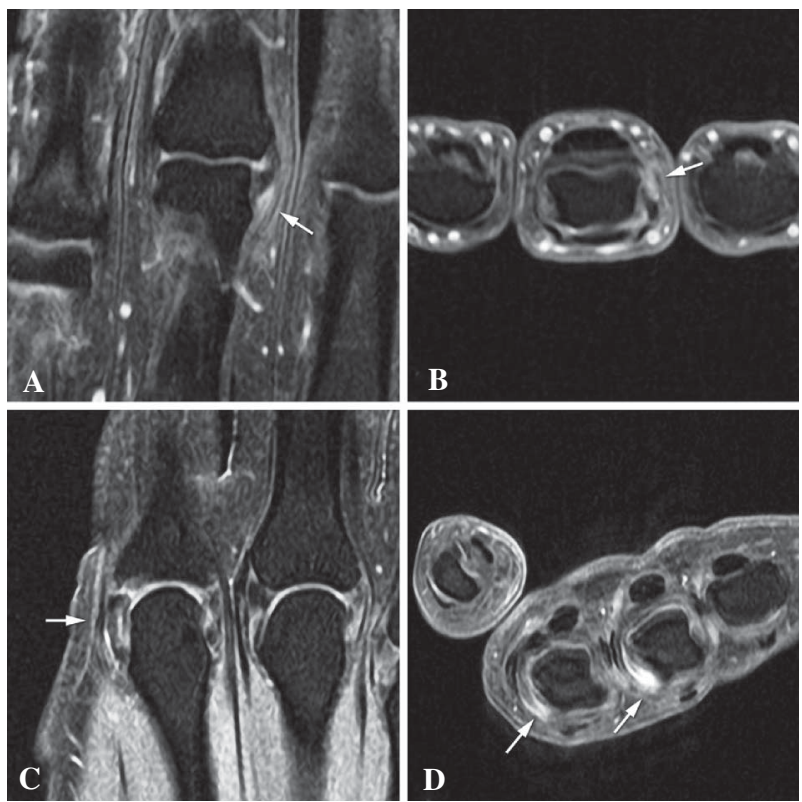

Figure 1. (A) Postgadolinium fat-suppressed coronal and (B) axial T1weighted images showing enthesitis next to the third PIP joint in one IBD patient with hand arthralgia and (C) postgadolinium fat-suppressed coronal and (D) axial T1-weighted images showing enthesitis next to the second and third MCP joints in another IBD patient with hand arthralgia.

Most studies in which imaging of the joints in IBD patients was performed have included patients with IBD associated with $\mathrm{SpA}$, in whom enthesitis is a very common finding $(3,4)$. Using ultrasound, Bandinelli et al (5) studied lower limb entheses in IBD patients without clinical signs and symptoms of SpA. The majority of their IBD patients had entheseal alterations (enthesis thickness, the presence of enthesophytes, bursitis, and erosion) while only a small minority of healthy controls had entheseal alterations. Power Doppler (PD) positive enthesitis was found in 13/ $81(16 \%)$ patients and in none of the healthy controls. Although a different imaging procedure and scoring system was used, these results are in line with ours.

The pathogenesis of musculoskeletal manifestations of IBD is not yet fully understood. Because of the shared pathogenesis of IBD and SpA, we expected to find enthesitis in the painful joints of IBD patients with arthralgia, and this was indeed the most commonly observed finding. It has been suggested that synovitis in SpA might develop secondary to enthesis (6).

In the control group, a small amount of bone marrow oedema was seen in the metacarpal heads of two patients, of which the clinical relevance is unknown. A limitation of the study is the small number of patients studied as a result of the selective inclusion criteria.

In conclusion, enthesitis was detected in this small group of IBD patients with arthralgia. Studies with a large number of patients are needed to obtain a good estimate of the prevalence of these findings on MRI and longer follow-up is needed to determine whether enthesitis on MRI will develop into clinically active enthesitis and arthritis.

\section{Acknowledgements}

The work of WS is supported by the Centre for Translational Molecular Medicine, The Netherlands.

\section{References}

1. Brakenhoff LKPM, van der Heijde DM, Hommes DW, Huizinga TWJ, Fidder HH. The joint-gut axis in inflammatory bowel diseases. J Crohns Colitis 2010;4:257-68.

2. Krabben A, Stomp W, van der Heijde DMFM, van Nies JAB, Bloem JL, Huizinga TWJ, et al. MRI of hand and foot joints of patients with anticitrullinated peptide antibody positive arthralgia without clinical arthritis. Ann Rheum Dis 2013;72:1540-4.

3. Emad Y, Ragab Y, Bassyouni I, Moawayh O, Fawzy M, Saad A, et al. Enthesitis and related changes in the knees in seronegative spondyloarthropathies and skin psoriasis: magnetic resonance imaging case-control study. J Rheumatol 2010;37:1709-17.

4. D'Agostino MA, Said-Nahal R, Hacquard-Bouder C, Brasseur JL, Dougados M, Breban M. Assessment of peripheral enthesitis in the spondylarthropathies by ultrasonography combined with power Doppler: a cross-sectional study. Arthritis Rheum 2003;48:523-33.

5. Bandinelli F, Milla M, Genise S, Giovannini L, Bagnoli S, Candelieri A, et al. Ultrasound discloses entheseal involvement in inactive and low active inflammatory bowel disease without clinical signs and symptoms of spondyloarthropathy. Rheumatology (Oxford) 2011; 50:1275-9.

6. McGonagle D, Gibbon W, Emery P. Classification of inflammatory arthritis by enthesitis. Lancet 1998;352:1137-40. 